subtotal deficiency of C6 and C7 was reported by $\mathbf{P}$. J. Lachmann (MRC Group on Mechanisms in Tumour Immunity, Cambridge). The proband is in good health. The deficiencies of both components segregate together in the grandchildren, which is to be expected if the structural genes for both proteins are involved, since they are closely linked. Trace C6 and C7 activity appear in the proband's serum in the same pattern of bands, different from either normal C6 or C7 when separated by isoelectric focusing. An interesting speculation is that this may be the result of a 'Lepore' fusion of $\mathrm{C} 6$ and $\mathrm{C} 7$ genes.

At times the Workshop veered from the strict limits of complement genetics, Alper and F. S. Rosen (Children's Hospital, Boston) presented evidence that $\mathrm{C} 3$ nephritic factor is an IgG, on the basis of functional detection of clonal electrophoretic variants and on the transmission of such a clone across the placenta. A. Ferreira (New York University) described a new C4binding protein in mice, the molecular association giving the appearance of $H-2$ linkage. Since more than half of the known complement components now have genetic markers, new components are needed if healthy growth of the topic is to be maintained!

\section{Progress in coronaviruses}

\section{from David J. Garves}

A symposium on Human and Animal Coronaviruses was held at the Agricultural Research Council Institute for Research on Animal Diseases, Compton UK on 24-25 March, 1977, and was organised by Professor A. P. Waterson, Royal Postgraduate Medical School, London, and Dr D. J. Garves, Compton.

THE Coronaviridae comprise a family of morphologically similar viruses that cause a wide variety of diseases in man and other animals. Following the recognition of coronaviruses as a distinct taxonomic group in 1968 (see Nature 220, 650), with virus particles having a peripheral halo in negatively stained preparations, the number of viruses classified in the group and our knowledge of their characteristics has greatly increased. There are still large gaps in our understanding of the diagnosis, pathogenesis, structure and serology of this economically and, potentially, medically important group and attempts were made to fill these gaps at the symposium.

Recent reports of coronavirus-like particles in samples from human and equine enteritis, human nephropathy and feline infectious peritonitis have raised the problem of which criteria should be used to identify a coronavirus. It has been found that coronaviruses are difficult to establish in cell culture and for this reason morphology of the virus particle provides the major distinguishing feature. J. Almeida (Wellcome Research Laboratories) and R. Bingham (Royal (Dick) School of Veterinary Studies, Edinburgh) reported new evidence for the morphology and structure of the internal component of avian infectious bronchitis virus. They showed electron micrographs of virus particles within which flask-shaped structures could be seen, the mouth of the 'flask' appearing to open at the outer membrane of the virus. Whether these structures are present in other coronaviruses and what relation they have to the organisation of the genetic material has yet to be determined.

Recent studies on the polypeptide structures of human and animal coronaviruses have shown that, while the number and size of the structural proteins vary between members of the family, some similar features can be seen. The polypeptide profiles may provide a method to distinguish relationships within the coronaviruses and in this context the apparent similarity between the polyacrylamide gel patterns for avian infectious bronchitis virus (M. R. Macnaughton, Clinical Research Centre, London) and calf diarrhoea coronaviruses (J. Laporte, Thiverval-Grignon, France) was of interest.

The genome of avian and porcine coronaviruses has been shown to comprise single-stranded RNA but whether this has a messenger role, as in oncornaviruses and enteroviruses, or is a complementary strand, as in paramyxoviruses, has not been determined. Our understanding of the strategy of the coronavirus genome is greater, however, following the reports of Macnaughton and D. H. Pocock (ARC, Compton) that avian and porcine coronavirus RNA contains polyadenylic acid tracts. This evidence strongly suggests that the RNA has a messenger function and is yet another feature, together with virion morphology and the genome size, that coronaviruses have in common with the RNA tumour viruses. Bingham reported, however, that attempts to detect a reverse transcriptase in avian infectious bronchitis virus have proved unsuccessful, in line with the evidence from inhibitor studies suggesting that coronavirus replication does not pro- ceed by way of DNA.

D. J. Alexander (Central Veterinary Laboratory, Weybridge) reported on the finding of long term infection of chickens with infectious bronchitis virus and these results, together with the evidence of chronic infection of mice with hepatitis virus and the carrier status of pigs with transmissible gastroenteritis, prompted K. McIntosh (University of Colorado, Denver) to suggest that future work on the human respiratory coronaviruses should consider implications of inapparent, chronic infections. It is possible that Balkan endemic nephropathy may result from such an infection, as $\mathbf{K}$. Apostolov (Royal Postgraduate Medical School, London) reported a possible serological relationship between this disease and a human respiratory coronavirus.

Coronavirus serology is confused at the moment, with cross reactions being detected between human, bovine, murine and porcine viruses. The serological relationship between porcine transmissible gastroenteritis virus and a canine enteric virus is strong and D. J. Reynolds, (Compton) also presented evidence of antibodies to transmissible gastroenteritis in cats. It is possible that the agent in the cat responsible for the very high antibody levels may be associated with feline infectious peritonitis, a possible coronavirus whose morphology and physicochemical properties were presented by A. Osterhaus (Utrecht, Netherlands). The morphology of the purified particles was not characteristic of other coronaviruses and whether this was due to degradation or a different surface projection structure is not known. The agent of feline infectious peritonitis demonstrated the problem caused by the fact that coronavirus identification rests principally on the shape of the surface projection. It was generally agreed that other features including virus structure, the characteristics of the genome and the mode of replication within the cell needed to be determined before any virus could be assigned to the Coronaviridae, although this information is not known for some well accepted coronaviruses. Similarly, more data would be required before any meaningful subdivisions, such as separating the avian from the mammalian members, could be made within the Family.

In the field of coronavirus disease control there seemed to be doubtful correlation between antibody levels in serum and secretions and resistance to infection. It was recommended that more effort should go into investigating cellular and humoral aspects of immunity to diseases caused by coronaviruses. 